INFLAMMATORY BOWEL DISEASE

\title{
Antibiotic use and the development of Crohn's disease
}

\author{
T Card, R F A Logan, L C Rodrigues, J G Wheeler
}

Gut 2004;53:246-250. doi: 10.1136/gut.2003.025239

See end of article for authors' affiliations .....................

Correspondence to: Dr T Card, University of Nottingham, Division of Epidemiology and Public Health, Queen's Medical Centre, Nottingham NG7 2UH, UK; tim.card@ nottingham.ac.uk

Accepted for publication 26 August 2003

\begin{abstract}
Background: Few environmental determinants of Crohn's disease are well established. Some observational data exist to implicate antibiotic use as a risk factor but these are derived from studies using questionnaires to assess reported antibiotic use that were susceptible to recall bias. We have therefore explored this relationship in prospectively gathered data.

Methods: We selected incident cases of Crohn's disease from the General Practice Research Database with at least five years of data prior to diagnosis. Controls with five years of complete data were randomly selected. Data were extracted on smoking, drug prescriptions, age, sex, and a variety of symptoms and diagnoses that might be indicative of occult Crohn's disease. Logistic regression was used to investigate the relationship between antibiotic use and Crohn's disease.

Results: A total of 587 Crohn's disease cases and 1460 controls were available for analysis. We found that antibiotic use $2-5$ years pre-diagnosis occurred in $71 \%$ of cases compared with $58 \%$ of controls $(p<0.001)$, and the median number of courses was two in the cases and one in the controls $(p<0.001)$. Adjusting for age, sex, smoking, and use of other drugs, antibiotic use had an odds ratio of 1.32 (1.051.65). We were unable to show specificity to any subgroup of antibacterials. Associations similar to that with antibiotics were also found with oral contraceptive, cardiovascular, and neurological drugs.

Conclusions: We found a statistically significant association between Crohn's disease and prior antibiotic use. This cannot be explained by recall bias, but due to lack of specificity it is unclear whether it is causal.
\end{abstract}

$\mathrm{T}$ he aetiology of Crohn's disease remains largely unknown. In recent years much attention has focused on genetic factors due to the well demonstrated heritability of the disease and the subsequent demonstration of genetic linkages first with areas of the genome and more recently with specific genes. ${ }^{1}$ Nevertheless, while genetic factors clearly play an important role, they cannot account for the rise in incidence of the disease over the last century. ${ }^{2}$ While many environmental factors have been proposed, smoking ${ }^{3}$ is the only one which is well established to date, but it is not sufficient to explain the entire environmental component of the causation of Crohn's disease. ${ }^{4}$ One aspect of the environment which is increasingly accepted as being associated with the aetiology of Crohn's disease is the intestinal microflora. ${ }^{5}$ If as is suggested by animal models such as the interleukin 10 deficient mouse, ${ }^{6}$ and the emerging evidence regarding probiotics, ${ }^{7}$ inflammatory bowel diseases may depend on the relationship between the gut and its microflora, then anything interfering with this relationship might contribute to their aetiology. Antibiotics can clearly alter at least temporarily the bacterial microflora and are therefore worth considering as a potential risk factor for inflammatory bowel disease.

The first evidence of the possibility that alterations in intestinal microflora, occasioned by antibiotics, might contribute to the aetiology of Crohn's disease was from two case control studies $^{89}$ which showed an association between increased antibiotic use and diagnosis of Crohn's disease in children. Both studies relied on recall of the use of antibiotics, assessed many years after the initial diagnosis. Such recall of exposures is known to be susceptible to bias in studies where the onset of disease could influence recall. In both studies the authors considered the finding an artefact, and not causal. Demling in $1994^{10}$ hypothesised on the basis of a subjective assessment of the trends in Crohn's disease and antibiotic use over time that a causal link may exist. As antibiotic use is common, it might make a large contribution to the risk of
Crohn's disease in the population if the association these studies have described is causal.

In order to examine the hypothesis that antibiotic use is associated with the subsequent development of Crohn's disease, while removing the potential for recall bias, we conducted a case control study using the General Practice Research Database (GPRD), in which data on antibiotics usage are recorded at the time of prescribing.

\section{METHODS}

The GPRD is a computerised database containing data on approximately 35 million patient years, gathered from general practices throughout the UK, and represents approximately $5 \%$ of the UK population. It is the largest such database in the world. The records within this database represent the observations, diagnoses made, and treatments prescribed or administered by general practitioners as well as those reported to them by hospital letters. The quality of the data is ensured by routine monitoring of the quality of information submitted by all contributing practices; data are coded as being "up to standard" when recording by practices has been verified to meet the quality criteria required (which includes the recording of at least $95 \%$ of consultations and diagnoses, including those made as a result of referral to secondary care). A number of evaluations have been made of the quality of the data in the GPRD, ${ }^{11}{ }^{12}$ and disease within the population included has been shown to be representative of disease within the whole UK population. ${ }^{12}$ The accuracy of the diagnosis of Crohn's disease within the GPRD has been specifically validated and found to be accurate in $94 \%$ of cases when rechecked via the general practitioner. ${ }^{13}$

\footnotetext{
Abbreviations: GPRD, general practice research database; PAF, population attributable fraction; BNF, British National Formulary; OR, odds ratio
} 


\section{Subjects}

All data used in this study were derived from a subset of the GPRD prepared for a different study (funded by the Department of the Environment, Transport, and the Regions to investigate a possible relationship between exposure to Mycobacterium paratuberculosis via drinking water and Crohn's disease).

The subset contained data from all subjects for whom a new diagnosis of Crohn's disease was recorded while they were contributing "up to standard" data to the GPRD. In addition, for each case, two individually age and sex matched controls, individuals with no previous diagnosis of Crohn's disease or ulcerative colitis, were available.

Cases with five or more years of prospectively gathered data prior to the first diagnosis of Crohn's disease were extracted. Cases for whom a diagnosis of ulcerative colitis had also been recorded were discarded. The controls used were a randomly selected sample of the full set of the controls who also had over five years of prospectively gathered data.

\section{Exposures}

Information on exposures extracted from the GPRD included age at index date (grouped in 20 year age bands), sex, smoking (classified as current smoking, non-smoking, or unknown based on the last relevant record in the dataset prior to the index date), all drugs prescribed, reason for consultations (usually a symptom or diagnosis), and the dates on which these occurred.

All events recorded as gastrointestinal infections, perianal sepsis, anaemia, irritable bowel syndrome, abdominal pain, or diarrhoea were identified among cases using a combination of Oxmis and Read codes. ${ }^{14}{ }^{15}$ These are referred to as gastrointestinal events. Each of these events was assigned to the six month period in which it occurred, working back from an index date which was the date of Crohn's disease diagnosis for cases and the date of the end of data for controls (that is, events were coded as occurring in the lst, 2nd, 3rd ..... nth six month period prior to the index date). In order to assess whether symptoms were consistent with undiagnosed Crohn's disease, the number of gastrointestinal events for each subject during each six month block was plotted (fig 1). Figure 1 shows an excess of events consistent with undiagnosed Crohn's disease among cases which was most marked in the last two years prior to diagnosis. Based on this finding we limited our primary analyses of antibiotic exposure to the period from the third to the fifth year prior to the index date, to reduce the possibility that antibiotic exposure followed the onset of any symptom or illness due to

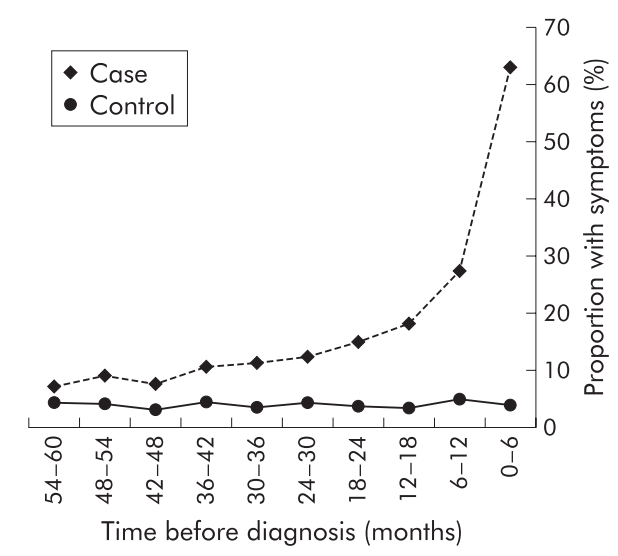

Figure 1 Proportion of cases and controls with symptoms consistent with undiagnosed Crohn's disease recorded within the six month period before the index date.
Crohn's disease. To further assess the possibility that any association between antibiotics and Crohn's disease might be due to prescriptions for the symptoms of undiagnosed Crohn's disease (that is, of reverse causality) we divided cases and controls according to whether, during the studied period (the third to fifth year prior to diagnosis), they had any record of gastrointestinal events, or had any drugs for gastrointestinal disease prescribed (gastrointestinal drugs).

Drugs prescribed were classified according to sections of the British National Formulary (BNF), which groups drugs by indication and by pharmacological type. For example, drugs used for infections are in chapter 5, antibacterials in section 5.1, and penicillins in section 5.1.1. Prescriptions were assigned to six month periods as described for the symptoms and diagnoses above. In this way we counted the prescriptions of antibiotics both in total and in the subgroups defined based on the subheadings in BNF section 5.1. We also counted all prescriptions, prescriptions of drugs for gastrointestinal disease (BNF chapter 1) as gastrointestinal drugs, oral contraceptive pills (BNF section 7.3.1 and 7.3.2) (previously described as associated with Crohn's disease), ${ }^{16}{ }^{17}$ drugs used for cardiovascular disease (BNF chapter 2), and drugs for disorders of the central nervous system (BNF chapter 4) (the last two as "control drugs", not expected to be associated with Crohn's).

\section{Analyses}

All analyses were conducted using Stata 7. We calculated odds ratios (OR) for developing Crohn's disease according to our main suspected exposure, usage of antibiotics $2-5$ years before diagnosis, and other exposures. Logistic regression was used to assess exposures and to control for potential confounders-that is, age, sex, and smoking. To correct for the effect of an individual's tendency to receive prescriptions in general (rather than those specifically for antibiotics) we included within the regression analyses a measure of the prescription of other drugs, as the total number of prescriptions minus the number for antibiotics. This was coded into quartiles. These analyses were repeated for cases with gastrointestinal symptoms and prescriptions in the 2-5 years before diagnosis (indicating possible undiagnosed Crohn's disease), and those subjects with no symptoms or gastrointestinal drugs during that period. Additionally, separate estimates were made for different groups of antibiotics, and for different frequencies of antibiotic prescriptions. To assess whether any association was specific to antibiotics as opposed to other drug groups, we repeated our analyses for oral contraceptive pills, drugs used for cardiovascular disease, and drugs for disorders of the central nervous system. Finally, to assess the potential importance of any association found if it were causal, we calculated the population attributable fraction (PAF) using appropriate methodology for multivariate analyses. ${ }^{18}$

\section{RESULTS}

A total of 587 cases and 1460 controls were available for analysis. Cases had a median of 8.1 years of data available (range 5.1-11.6) of which 6.4 years (range 5.0-10.5) occurred before diagnosis. Controls had a median of 8.2 years of data (range 5.0-11.6). Cases and controls had a similar sex distribution (43\% male for cases and $44 \%$ for controls), but cases were slightly older, with a mean age at the index date 2.5 years greater than that of controls (table 1). A greater proportion of cases were recorded as being current smokers (28\% $v 20 \%)$. Antibiotics were prescribed on at least one occasion $2-5$ years before the index date for $71 \%$ of cases and $58 \%$ of controls $(\mathrm{p}<0.001)$, and cases received on average a greater total number of antibiotic prescriptions (median 2 compared with 1 for controls). 
Table 1 Age, sex, antibiotic use, date of entry to study, and smoking in cases and controls

\begin{tabular}{|c|c|c|c|}
\hline & Cases $(n=587)$ & Controls $(n=1460$ ) & \\
\hline & n (\%) & n (\%) & p Value \\
\hline \multicolumn{4}{|l|}{ Age (y) } \\
\hline $0-20$ & $79(13)$ & $76(5)$ & $<0.001$ \\
\hline $20-40$ & 207 (35) & 569 (39) & \\
\hline $40-60$ & $156(27)$ & $445(30)$ & \\
\hline $60-80$ & $145(25)$ & $370(25)$ & \\
\hline Median & 41.64 & 43.76 & 0.003 \\
\hline \multicolumn{4}{|l|}{ Sex } \\
\hline Male & $253(43)$ & $642(44)$ & 0.7 \\
\hline Female & $334(57)$ & $818(56)$ & \\
\hline \multicolumn{4}{|c|}{ Date of entry to study } \\
\hline Median & $1 / 10 / 90$ & $1 / 03 / 90$ & $<0.001$ \\
\hline Range & $1 / 06 / 87-23 / 08 / 93$ & $1 / 06 / 87-8 / 10 / 93$ & \\
\hline \multicolumn{4}{|l|}{ Smoking } \\
\hline Smoker & $165(28)$ & $297(20)$ & 0.001 \\
\hline Non-smoker & $291(50)$ & 796 (55) & \\
\hline Unknown & $131(22)$ & $367(25)$ & \\
\hline \multicolumn{4}{|c|}{ Antibiotic courses } \\
\hline At least one & 418 (71) & $843(58)$ & $<0.001$ \\
\hline Median & 2 & 1 & $<0.001$ \\
\hline
\end{tabular}

In a multiple logistic regression analysis of all cases, there were statistically significant associations between Crohn's disease and prescription of antibiotics 2-5 years before the index date (OR 1.32 (95\% confidence interval (CI) 1.05$1.65)$ ), other drugs (OR 1.54 (95\% CI $1.38-1.70)$ ), and smoking (OR 1.55 (95\% CI 1.22-1.98) compared with nonsmokers) after adjusting for age and sex (table 2). Although sex and smoking were included in this model on an a priori basis, they did not appreciably confound the relationship between antibiotics and Crohn's disease (their omission from the model leaving the OR for antibiotic use unchanged at 1.32). Restricting analysis to cases with no symptoms or gastrointestinal drugs, suggestive of undiagnosed Crohn's disease for 2-5 years before diagnosis (252 cases and 1022 controls), the association with antibiotic prescriptions was slightly stronger (OR 1.53 (95\% CI 1.12-2.07)), and was weaker with other drugs prescribed (OR 1.19 (95\% CI 1.03-1.08)).

Examination of the associations between Crohn's disease and different groups of antibiotics and with differing levels of exposure gave ORs ranging between 0.95 and 1.71 (table 3). For two groups the OR was significantly greater than 1

Table 2 Multivariate logistic regression models* of the association between antibiotic use and subsequent diagnosis of Crohn's disease

\begin{tabular}{lll}
\hline & OR & $95 \% \mathrm{Cl}$ \\
\hline All subjects & & \\
Smoker & 1.55 & $1.22-1.98$ \\
Smoking status unknown & 0.86 & $0.64-1.16$ \\
Drugs other than antibiotics $†$ & 1.54 & $1.38-1.70$ \\
$\quad$ Antibiotics $\neq$ & 1.32 & $1.05-1.65$ \\
Subjects with no symptoms or GI & & \\
drugs 3-5 y pre-diagnosis only & & \\
Smoker & 1.55 & $1.08-2.20$ \\
Smoking status unknown & 0.79 & $0.52-1.18$ \\
Drugs other than antibiotics & 1.19 & $1.03-1.38$ \\
Antibiotics & 1.53 & $1.12-2.07$ \\
\hline
\end{tabular}

OR, odds ratio; $95 \% \mathrm{Cl}, 95 \%$ confidence interval.

*All models include age and sex as cofactors in addition to those listed. †Drugs other than antibiotics represents prescriptions for all other drugs with the exception of antibiotics, and the OR is for moving from one quartile of prescription density to the next.

fFor antibiotics the OR is for a comparison of any prescription for that group against none. (tetracyclines: OR 1.33 (95\% CI 1.01-1.77) and metronidazole and tinidazole: OR 1.71 (95\% CI 1.05-2.76)). Restriction of the analysis to subjects who had no symptoms suggestive of undiagnosed Crohn's disease and no prescriptions for gastrointestinal drugs recorded $2-5$ years before the index date rendered the association with metronidazole and tinidazole non-significant (OR 1.08 (95\% CI $0.39-2.99$ )) although the association with tetracycline strengthened. There was also no evidence of a dose response relationship between the frequency of any antibiotic prescription and Crohn's disease, with similar ORs for 1, 2-5, or more than 5 courses at 2-5 years prior to the index date (table 4).

When our analysis was repeated to examine drug groups other than antibiotics, we found similar associations between other drugs and Crohn's disease. Among all cases ORs of 1.34 (1.08-1.66), 1.25 (0.95-1.66), and 1.48 (1.00-2.17) were found, respectively, for neurological drugs, cardiovascular drugs, and oral contraceptives in multivariate analyses analogous to those presented in (table 3 ). These associations however did not remain significant in the subset analysis examining subjects without possible symptoms of Crohn's disease or gastrointestinal drugs (table 5).

Based on the observed OR of 1.32 for any antibiotic exposure and the observed $81 \%$ of the cases using antibiotics over the period 2-5 years prior to diagnosis, the calculated PAF was $17 \%$. The equivalent figure for smoking was $10 \%$.

\section{DISCUSSION}

This study is the first to examine in detail antibiotic use as a risk factor for Crohn's disease and the first to assess it in adults. It provides evidence of an association between antibiotic use and the subsequent diagnosis of Crohn's disease. A variety of factors suggest that this association is real but a lack of specificity suggests that its causality is uncertain. Our study also throws some light on the previously described association between Crohn's disease and the use of the oral contraceptive pill as although we have replicated this result, similar questions are raised as to whether this association is causal.

The use of the GPRD to carry out this study confers a number of benefits. Firstly, unlike the two previous studies in which antibiotic use was ascertained after the diagnosis of Crohn's disease, ${ }^{8}{ }^{9}$ this study utilised data on antibiotic use 
Table 3 Effect of different groups of antibiotics. All odds ratios are corrected for age, sex, smoking, and prescriptions of all non-antibacterial drugs

\begin{tabular}{|c|c|c|c|c|c|c|c|c|}
\hline \multirow[b]{3}{*}{ Group } & \multicolumn{4}{|l|}{ All subjects } & \multicolumn{4}{|c|}{ Subjects with no symptoms or GI drugs } \\
\hline & \multicolumn{2}{|l|}{ No exposed } & \multirow[b]{2}{*}{ OR } & \multirow[b]{2}{*}{$95 \% \mathrm{Cl}$} & \multicolumn{2}{|l|}{ No exposed } & \multirow[b]{2}{*}{ OR } & \multirow[b]{2}{*}{$95 \% \mathrm{Cl}$} \\
\hline & Cases $(n=587)$ & Controls $(n=1460)$ & & & Cases $(n=252)$ & Controls $(n=1022)$ & & \\
\hline $\begin{array}{l}\text { Ben pen, penicillinase resistant } \\
\text { pens }\end{array}$ & 128 & 286 & 0.94 & $0.74-1.21$ & 47 & 169 & 0.93 & $0.64-1.36$ \\
\hline $\begin{array}{l}\text { Broad spectrum and } \\
\text { anti-pseudomonal penicillins }\end{array}$ & 271 & 531 & 1.10 & $0.90-1.36$ & 96 & 317 & 1.04 & $0.77-1.42$ \\
\hline Cephalosporins & 91 & 153 & 1.18 & $0.88-1.58$ & 29 & 78 & 1.30 & $0.81-2.09$ \\
\hline Tetracyclines & 98 & 172 & 1.34 & $1.01-1.77$ & 36 & 91 & 1.72 & $1.12-2.64$ \\
\hline Macrolides & 87 & 188 & 0.96 & $0.72-1.27$ & 32 & 95 & 1.12 & $0.71-1.75$ \\
\hline Sulphonamides and trimethoprim & 87 & 160 & 1.16 & $0.86-1.57$ & 21 & 68 & 1.21 & $0.71-2.05$ \\
\hline Metronidazole and tinidazole & 33 & 45 & 1.70 & $1.05-2.75$ & 5 & 19 & 1.08 & $0.39-2.99$ \\
\hline Quinolones & 31 & 55 & 1.15 & $0.72-1.83$ & 4 & 19 & 0.67 & $0.22-2.03$ \\
\hline Other & 8 & 17 & 1.09 & $0.46-2.57$ & 2 & 5 & 1.66 & $0.31-8.76$ \\
\hline
\end{tabular}

which were collected before the diagnosis was made. This establishes clearly that the associations found in previous studies are unlikely to have resulted solely from bias with respect to recall of antibiotic use. Recall bias cannot explain any of the associations found in this study. In addition, the use of a complete cohort of eligible patients from a large representative database and an appropriate group of controls makes selection bias unlikely.

Therefore, if the association was not the result of bias, could it be due to confounding? There are a few known risk factors for Crohn's disease, with age, sex, smoking, and family history being the most important. Of these we have data on all but family history and have therefore been able to construct a multivariate model correcting for the effects of age, sex, and smoking. We were unable to adjust for possible confounding by family history although for this to be a confounder it would need to be associated with antibiotic use, which seems unlikely. The data available on smoking are incomplete and it is possible that some confounding remains. However, as the effect of adjusting for smoking classified as smoker/non-smoker was so small, it seems highly unlikely that the associations demonstrated are explained by residual confounding by smoking.

We considered two other potential artefacts: reverse causation and a possible "tendency to receive prescriptions". A challenge for any study examining the aetiology of a chronic disease is to define the date of onset accurately, and hence to establish whether exposures preceded the onset of symptoms. This is necessary to exclude reverse causation, the situation when an exposure is in fact a response to symptoms of the still undiagnosed disease. Although the date of diagnosis of inflammatory bowel diseases has been validated within the GPRD and found to be accurate to within a few weeks, ${ }^{13}$ it is likely that for a proportion of patients the time between onset of symptoms and diagnosis can be long, as shown by the analysis in fig 1 . Thus it remains possible that the association seen may at least in part be due to antibiotics prescribed as treatment for the symptoms of undiagnosed Crohn's disease. We corrected for this by firstly excluding from our multivariate analyses the two year period before diagnosis in which symptoms were common; and secondly by repeating all analyses in the subgroup of cases with no gastrointestinal symptoms or prescription of gastrointestinal drugs within the period $2-5$ years prior to diagnosis. The fact that the association between antibiotics and Crohn's disease remains, and is of the same magnitude in the subgroup analysis, is evidence against the association being a result of reverse causation.

To correct for the tendency of subjects to receive prescriptions, we explored whether the association between diagnosis of Crohn's disease and prescription of antibiotics was specific in three ways. Firstly, we examined whether the association was limited to specific groups of antibiotics. Although the power available for this analysis was limited, we were unable to demonstrate a clear specificity with any antibiotic group as although only tetracyclines were found to have a significant odds ratio as a subgroup, the confidence interval for this odds ratio overlapped those of all other groups. Secondly, we examined whether the association was specific to antibiotics as opposed to other drug groups. We found an association similar to that for antibiotics with the oral contraceptive pill (this association is of similar magnitude to that previously described ${ }^{16}{ }^{17}$ ), but also found similar associations with cardiovascular and neurological drug prescribing. In each of these cases the association was close to the same magnitude but statistically non-significant (in contrast with the significant association with antibiotic use). This to some extent reflects the frequency of antibiotic use and hence the greater power to find associations for this drug group. Finally, we included in the multivariate model as a factor the number of prescriptions for drugs other than antibiotics, and from this there was evidence of some degree of confounding: the association between antibiotic use and Crohn's decreased from 1.56 to 1.32 when prescriptions for other drugs were

Table 4 Adjusted odds ratios for the relationships between different numbers of courses of antibiotics and Crohn's disease in subjects with no symptoms or gastrointestinal drugs

\begin{tabular}{|c|c|c|c|c|}
\hline $\begin{array}{l}\text { No of courses of } \\
\text { antibiotics }\end{array}$ & Cases $(n=252)$ & Controls ( $n=1022$ ) & OR & $95 \% \mathrm{Cl}$ \\
\hline $\begin{array}{l}1 \\
2-5\end{array}$ & $\begin{array}{l}62 \\
81\end{array}$ & $\begin{array}{l}192 \\
258\end{array}$ & $\begin{array}{l}1.57 \\
1.50\end{array}$ & $\begin{array}{l}1.07-2.29 \\
1.05-2.14\end{array}$ \\
\hline Over 5 & 20 & 50 & 1.51 & $0.82-2.78$ \\
\hline
\end{tabular}

OR, odds ratio; $95 \% \mathrm{Cl}, 95 \%$ confidence interval. 
Table 5 Multivariate regression models for oral contraceptive, cardiovascular, and central nervous system drugs compared with antibacterials

\begin{tabular}{|c|c|c|c|c|c|c|c|c|}
\hline \multirow[b]{3}{*}{ Group } & \multicolumn{4}{|c|}{ All subjects } & \multicolumn{4}{|c|}{ Subjects with no symptoms or GI drugs } \\
\hline & \multicolumn{2}{|c|}{ No exposed } & \multirow[b]{2}{*}{ OR } & \multirow[b]{2}{*}{$95 \% \mathrm{Cl}$} & \multicolumn{2}{|c|}{ No exposed } & \multirow[b]{2}{*}{ OR } & \multirow[b]{2}{*}{$95 \% \mathrm{Cl}$} \\
\hline & $\begin{array}{l}\text { Cases } \\
(n=587)\end{array}$ & $\begin{array}{l}\text { Controls } \\
(n=1460)\end{array}$ & & & $\begin{array}{l}\text { Cases } \\
(n=252)\end{array}$ & $\begin{array}{l}\text { Controls } \\
(n=1022)\end{array}$ & & \\
\hline Antibacterials & 418 & 843 & 1.32 & $1.05-1.65$ & 163 & 500 & 1.53 & $1.12-2.07$ \\
\hline Drugs for cardiovascular disease & 127 & 260 & 1.26 & $0.95-1.66$ & 41 & 139 & 1.49 & $0.97-2.29$ \\
\hline Drugs for nervous system disease & 275 & 520 & 1.34 & $1.08-1.66$ & 70 & 274 & 0.93 & $0.67-1.29$ \\
\hline Oral contraceptive pills & 94 & 198 & 1.48 & $1.00-2.17$ & 37 & 121 & 1.42 & $0.81-2.47$ \\
\hline
\end{tabular}

$\mathrm{OR}$, odds ratio; $95 \% \mathrm{Cl}, 95 \%$ confidence interval; $\mathrm{Gl}$, gastrointestinal.

included in the model. This adjustment however did not remove all of the association between antibiotic use and the subsequent diagnosis of Crohn's disease.

Both the lack of specificity and lack of a dose response relationship provide some evidence that the association between Crohn's disease and prior antibiotic use may not be causal. This begs the question of what alternative explanations there might be. Clearly, it is possible that despite our best efforts to exclude reverse causality our findings might be explained if Crohn's disease does indeed have a longer than generally recognised prodrome, which presents non-specifically to clinicians and hence causes a wide variety of prescriptions. Equally, it may be that only a proportion of cases of Crohn's disease are diagnosed, and that the probability of being diagnosed is related to the degree of contact the sufferer has with medical services (and hence the number of prescriptions which they receive). Finally, it is possible that some prescribed drug does indeed have a causal relationship with Crohn's disease, and that since the prescription of one drug is associated with the prescription of others, the association we have seen with each drug group is in effect a proxy for the association with this causally related drug.

Although caution needs to be exercised before accepting the hypothesis that there is a causal association between antibiotic use and Crohn's disease, it is equally important to guard against the premature rejection of the hypothesis. Despite its advantages, the dataset we have used for this study has allowed us only to examine the effect of recent use of antibiotics. There were very few subjects for whom much more than five years of data prior to diagnosis were available, and almost none with complete data from birth to diagnosis. We were therefore unable to examine the possibility that a greater and perhaps more specific effect would be seen from antibiotic use extending over a longer or earlier period or at a particular period of life. One potentially important period would be early childhood to which the previous studies of this association relate. ${ }^{8}$ 9

As a byproduct of this study, we have been able to examine the relationship between Crohn's disease and prior use of oral contraceptives. Several previous studies have reported odds ratios of $0.7-2.5$, with a meta-analysis giving a pooled OR corrected for confounding by smoking of 1.44 (1.12-1.86). ${ }^{19}$ These studies have in general, like previous antibiotic studies, been based on patient recall of exposure, and none has looked as we have at exposure to other drugs. Our finding of an OR of 1.48 in our corrected model for oral contraceptive use is consistent with these previous findings, but the lack of specificity that we have shown must also cast doubt on its causality in our study and in previous studies.

In conclusion, we have demonstrated a significant association between antibiotic use and diagnosis of Crohn's disease 2-5 years later in prospectively collected data. That we have not demonstrated a specific association does not disprove the hypothesis that antibiotics can contribute to the causation of
Crohn's disease. In view of the potential importance of the association with antibiotics (that if causal it might account for $17 \%$ of Crohn's disease), and the possibility that the time period available for study may not be the most appropriate, further study of this question is clearly required.

\section{ACKNOWLEDGEMENTS}

Dr Card is supported by a Wellcome Research Training Fellowship in Clinical Epidemiology (No 060529).

\section{Authors' affiliations}

T Card, R F A Logan, Division of Epidemiology and Public Health, University of Nottingham, Nottingham, UK

L C Rodrigues, London School of Hygiene and Tropical Medicine, London, UK

J G Wheeler, London School of Hygiene and Tropical Medicine, London, UK, and Department of Public Health and Primary Care, University of Cambridge, Cambridge, UK

\section{REFERENCES}

1 Bonen DK, Cho JH. The genetics of inflammatory bowel disease. Gastroenterology 2003;124:521-36.

2 Logan RF. Inflammatory bowel disease incidence: up, down or unchanged? Gut 1998;42:309-11.

3 Fiocchi C. Inflammatory bowel disease: etiology and pathogenesis. Gastroenterology 1998;115:182-205.

4 Tysk C, Lindberg E, Jarnerot G, et al. Ulcerative colitis and Crohn's disease in an unselected population of monozygotic and dizygotic twins. A study of heritability and the influence of smoking. Gut 1988;29:990-6

5 Podolsky DK. Inflammatory bowel disease. N Engl J Med 2002;347:417-29.

6 Sellon RK, Tonkonogy S, Schultz M, et al. Resident enteric bacteria are necessary for development of spontaneous colitis and immune system activation in interleukin-10-deficient mice. Infect Immun 1998;66:5224-31.

7 Jonkers D, Stockbrugger R. Probiotics and inflammatory bowel disease. J R Soc Med 2003;96:167-71.

8 Wurzelmann JI, Lyles CM, Sandler RS. Childhood infections and the risk of inflammatory bowel disease. Dig Dis Sci 1994;39:555-60.

9 Gilat T, Hacohen D, Lilos P, et al. Childhood factors in ulcerative colitis and Crohn's disease. An international cooperative study. Scand J Gastroenterol 1987;22:1009-24.

10 Demling L. Is Crohn's disease caused by antibiotics? Hepatogastroenterology 1994;41:549-51.

11 Walley T, Mantgani A. The UK General Practice Research Database. Lancet 1997:350:1097-9

12 Hollowell J. The General Practice Research Database: quality of morbidity data. Popul Trends 1997;87:36-40.

13 Lewis JD, Brensinger C, Bilker WB, et al. Validity and completeness of the General Practice Research Database for studies of inflammatory bowel disease. Pharmacoepidemiol Drug Saf 2002;11:211-18.

14 Chisholm J. The Read clinical classification. BMJ 1990;300:1092.

15 Lis Y, Mann RD. The VAMP Research multi-purpose database in the U.K. J Clin Epidemiol 1995;48:431-43.

16 Corrao G, Tragnone A, Caprilli R, et al. Risk of inflammatory bowel disease attributable to smoking, oral contraception and breastfeeding in Italy: a nationwide case-control study. Cooperative Investigators of the Italian Group for the Study of the Colon and the Rectum (GISC). Int J Epidemiol 1998;27:397-404

17 Persson PG, Leijonmarck CE, Bernell O, et al. Risk indicators for inflammatory bowel disease. Int J Epidemiol 1993;22:268-72.

18 Greenland S. Application of stratified analysis methods. In: Greenland S, ed. Modern epidemiology. New York: Lippincott, Williams and Wilkins, 1998:281-300.

19 Godet PG, May GR, Sutherland LR. Meta-analysis of the role of oral contraceptive agents in inflammatory bowel disease. Gut 1995;37:668-73. 